\title{
The implementation of new digital technologies during pre-shift examinations of personnel at energy enterprises
}

\author{
Rob. N. Khizbullin ${ }^{1}$, B.V. Chuvykin ${ }^{2}$, Rad.N. Khizbullin ${ }^{1}$, and A.A. Makhov ${ }^{1}$ \\ ${ }^{1}$ Kazan State Power Engineering University, Kazan, Russia \\ ${ }^{2}$ Penza State University, Penza, Russia
}

\begin{abstract}
The article discusses the possibility of using a new digital automated medical hardware complex which ensures health control of energy enterprise personnel. The considered complex consists of separate functional devices. Thanks to their technical characteristics a high reliability of the registered physiological parameters of the examined workers can be obtained. Thus, we get high reliability of personnel health monitoring. The automated complex carries out digital data processing. According to this data the prognostic state of the employee is issued. It plays a key role in improving safety at electric power enterprises, reduces the risk of accidents caused by personnel.
\end{abstract}

\section{Introduction}

The development of technology is currently impossible without the use of digital technologies. Digital transformation also covers the areas that were considered as conservative such as medicine with its invasive methods. The proposed digital automated medical hardware complex uses non-invasive optical methods based on the Bouguer and Bouguer-Lambert laws. This digital medical hardware complex is primarily intended for medical institutions. It can also be used in medical offices of energy enterprises during pre-shift examination of personnel according to legal requirements. A distinctive feature of the complex is microprocessor processing of physiological response signals.

Operations and repairs of energy facilities and equipment can be life-threatening. Personnel dealing with secure and dangerous energy facilities, as well as electrical and high-altitude work, may be under psychophysical influences. It affects the general physiological state of the employee during the work shift.

In the Russian Federation there are documents that regulate measures to ensure the reliability of production activities and preserve the health of workers: "Regulations on the psychophysical assurance of the reliability of professional activities and the health preservation of personnel of energy enterprises" of RAO UES of Russia dated 06/18/2000. This document defines the organizational and legal basis for the psychological support of the reliability of professional activities and the preservation of the health of employees. The psychophysical support is carried out due to the results of the surveys, including pre-shift examinations of personnel.
There is an Order of the Ministry of Energy of the Russian Federation , August 31, 2011 N 390 "On approval of the Procedure for medical examinations of workers dealing with the maintenance of electric power facilities"

There is a part 3 of Article 28 of the Federal Law of March 26, 2003 N 35-FZ "On the Electric Power Industry" which determines the procedure for medical examinations (survey) of employees directly dealing with the maintenance of energy facilities

In accordance with this Order at the request of the employee pre-shift medical examinations can be carried out to establish the fact of the use of alcohol, drugs or psychotropic substances and the psychophysical state of the personnel. There are mandatory items - measurement of pressure, pulse, body temperature.

There is a similar Order of the Ministry of Health and Social Development of Russia dated 04/12/2011 No. $302 n$ "On the approval of lists of harmful and (or) hazardous production factors and works, during which preliminary and periodic medical examinations (survey) are carried out, and the Procedure for conducting preliminary and periodic medical examinations (surveys) of workers employed in heavy work and with harmful and (or) dangerous working conditions."

In the Order of the Ministry of Health of Russia dated November 8, 2012, On the approval of the procedure for carrying out pre-shift (pre-trip) and postshift (post-trip) medical examinations in accordance with paragraph 7 of article of the Federal Law dated November 21, 2011 No. 323-FZ "On the basics of protecting the health of citizens in the Russian Federation" the procedure for carrying out pre-shift (pretrip) medical examinations is indicated, the first paragraph of which indicates: "Pre-shift (pre-trip) medical examinations are carried out before the start of a 
work shift (flight) in order to identify signs of exposure to harmful and (or) hazardous production factors, conditions and diseases that impede the performance of work duties, including alcohol, drug or other intoxication and residual effects of such intoxication."

To fulfill the requirements of the above orders, medical and technical support is necessary, sufficient to carry out pre-shift examination not more than 30 minutes before the start of the work shift $[3,4]$. Thus, it is necessary to quickly and accurately determine the state of health of a certain number of personnel, in a short period of time and give a prognostic conclusion of the physiological state of a person for a work shift.

\section{Solution of an urgent problem and practical implementation}

To help doctors in performing control over the physical state of persons at pre-shift examination at power facilities, an automated medical complex has been developed. This computerized hardware complex (Fig. 1) consists of the following main functional devices: a precision medical thermometer, a two-channel photoplethysmograph, and in the future it will be equipped with a three-channel pulse oximeter.

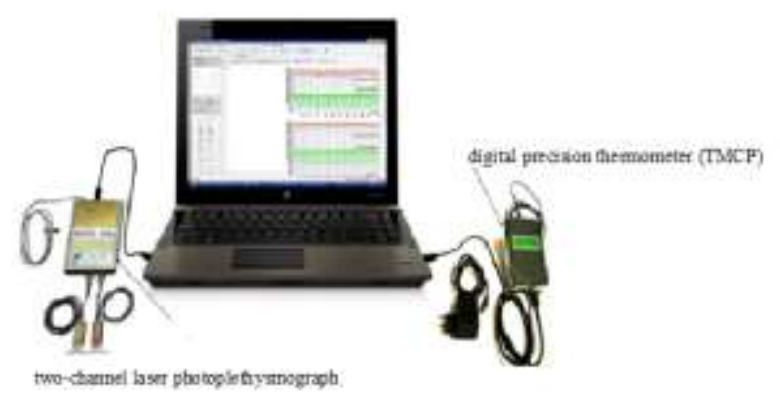

Fig. 1. Computerized hardware complex.

The main task of the developed automated medical complex for pre-shift examination (survey) is the identification with high reliability of the psychophysical state of the employee, as well as the predictability of his state during the work shift period. At the same time, the medical worker must quickly and accurately make a decision about permission to work and post-shift examination of the employee. All data for each examination are recorded in the database of pre-shift examinations, which is stored electronically.

As specified in the regulatory documents, the mandatory procedures for the examination are taking indicators of temperature, pressure, pulse. In the developed automated complex the measurement of temperature, pressure, pulse can be carried out simultaneously, which reduces the time of the examination.

In medicine, temperature is an important physiological parameter closely related to the physiological processes in the body. Therefore, temperature measurement in medical diagnostics is a mandatory procedure.
The temperature range of measurement of the developed medical digital precision thermometer (TMCP) is $+5 \ldots+50^{\circ} \mathrm{C}$, the accuracy is within $0.05 \ldots$ $0.1{ }^{\circ}$ C. For a high-quality visual presentation of temperature measurements, and for relative measurements, as well as for graphical display of temperature changes, it is important to have a high linearity and resolution of the measuring technical system. Temperature resolution should not exceed $0.01^{\circ}$ C.

Qualitative registration of temperature change in the body can be provided only by a low-inertia system. Its inertness should be at least an order of magnitude less than the temperature inertness of the measured area of the body. It means that the temperature sensor should have the smallest possible overall dimensions and thermal inertia.

Sensors must allow you to register temperature with an accuracy of $0.1^{\circ} \mathrm{C}$, have the smallest overall dimensions and the highest temperature sensitivity in the range of $+5 \ldots+50^{\circ} \mathrm{C}$. So, the thermistors are most widely used in medicine [1].

The general technical requirements for medical thermometers [2-4], [7,8] are fully met by the digital precision thermometer described in [5]:

- range of measured temperature $+5 \ldots+50^{\circ} \mathrm{C}$;

- the relative error of the device when measuring the temperature of $0.1 \%$;

-type of the used temperature sensor - thermistor;

- the absolute error of temperature measurement by an uncalibrated thermistor in the range $+25 \ldots+50^{\circ} \mathrm{C}$ is $0.1^{\circ} \mathrm{C}$ (additional calibration is possible);

-measurement rate-15 values per second.

The developed medical digital precision thermometer (TMCP) (Fig. 2), included in the automated complex, consists of two main parts: a temperature recording unit and a temperature sensor with a remote sensing element (thermal sensor) - thermistor. This thermal sensor allows you to register temperature with an accuracy of $\pm 0.1^{\circ} \mathrm{C}$.

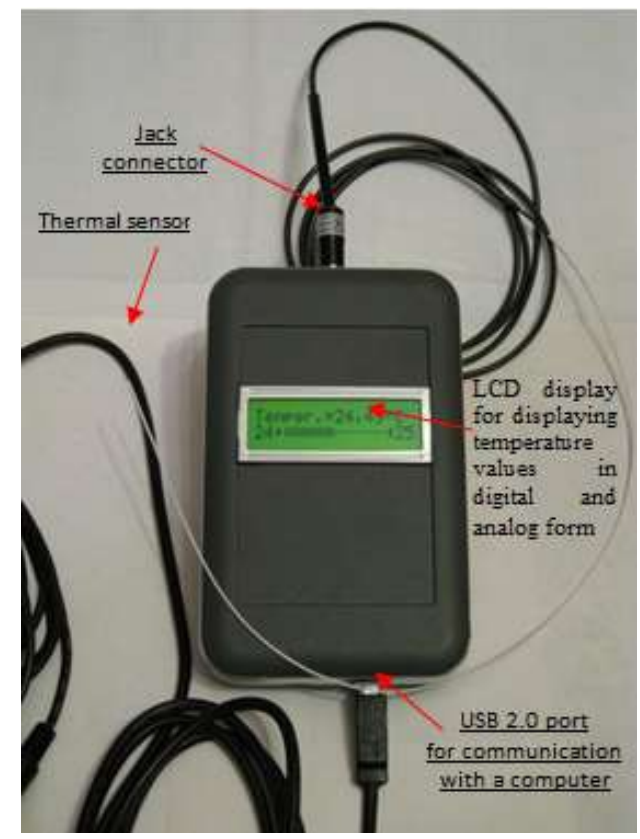

Fig. 2. Medical digital precision thermometer (TMCP). 


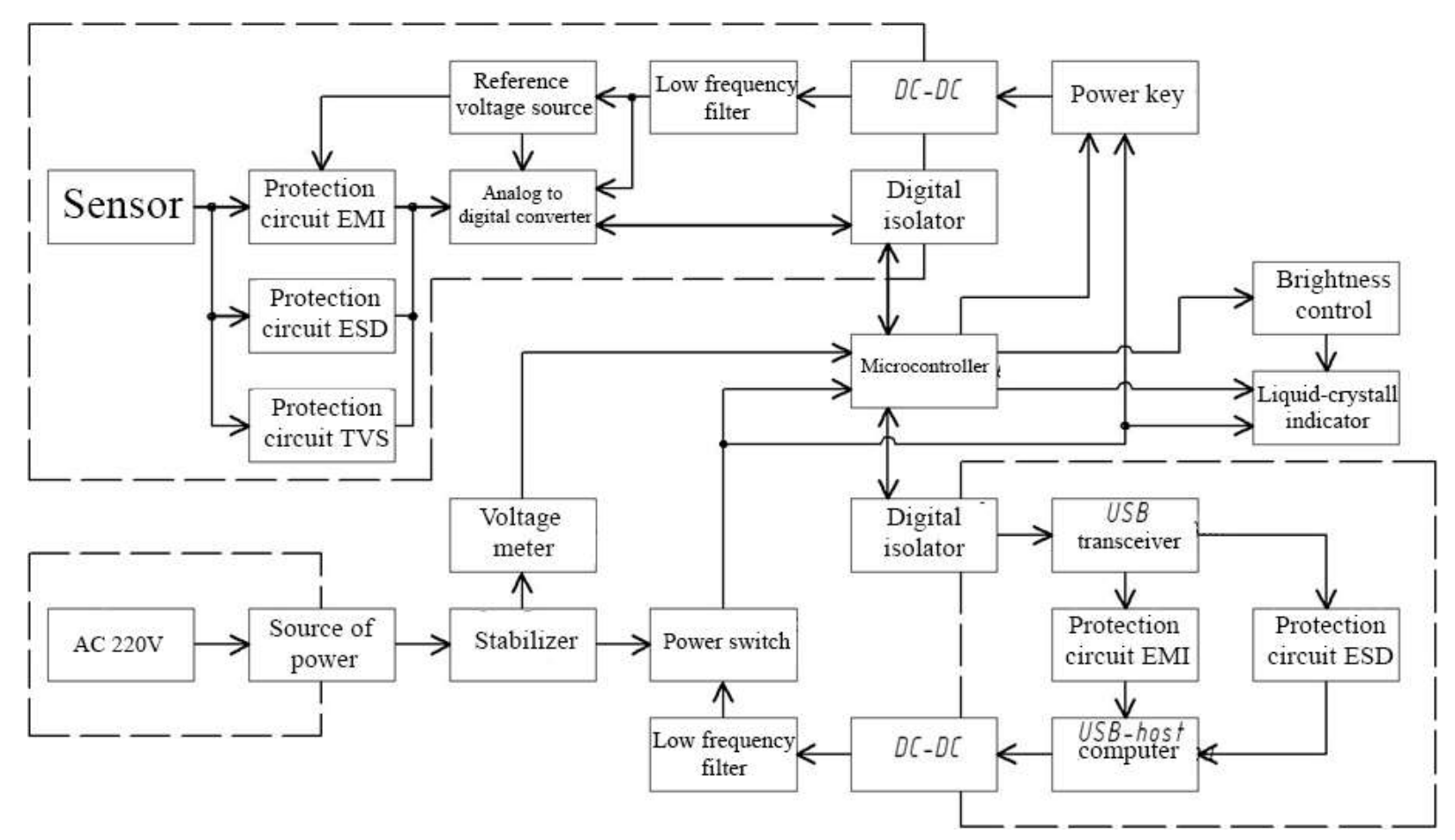

Fig. 3. Block diagram of the TMCP thermometer.

Length of the active part of the sensor is $450 \mathrm{~mm}$, diameter $1 \mathrm{~mm}$, diameter thermistor head $1.3 \mathrm{~mm}$. The sensor is connected with the unit using a JACK $6,35 \mathrm{~mm}$ connector. The total length of the transducer, including lead cable and connector, is $1.5 \mathrm{~m}$. The transducer can be sterilized using any common hospital disinfectant such as ethanol, isopropyl or chlorite compounds.

The registration unit processes the sensor signal and displays it on the screen. The main unit can be powered by either a USB 2.0 interface or an external medical power supply (a special adapter with a constant supply voltage in the range of $+7.5 \ldots+12 \mathrm{~V})$. When the unit is connected via USB 2.0, the temperature data is transferred to a computer and, by means of specialized software, is displayed on the monitor in real time. It is saved in the computer memory as a simple text file.

Block diagram of the thermometer is shown in Fig. 3 [6]. The signal from the temperature sensor goes to the amplifier, then to the analog-to-digital converter (ADC), where it is digitized and converted into a digital code, which is transmitted to the microcontroller through galvanic isolation. The microcontroller processes the received data, calculates the temperature value, displays the received information on the display and transmits the temperature data into a digital code via the galvanicaly isolated USB 2.0 interface to the computer. A power selector is also included into the circuit.

The second device of the automated complex is a two-channel photoplethysmograph, which functions on the basis of the method of digital photoplethysmography.

The use of digital photoplethysmography is of great diagnostic value in the quick and correct assessment of local capillary blood flow, measurement of blood pressure, pulse, blood flow velocity, vascular tone, heart rate, minute and systolic blood volume. Digital photoplethysmography quickly provides accurate and objective information about changes in blood circulation parameters under various physical factors. It can be effectively used in assessing the physiological state of technical personnel and workers at production facilities of the power industry, where pathogenic factors affect the human body. The diagnostic capabilities of photoplethysmography make it possible to predict the permissible dose of a pathogenic physical factor of influence. We can prevent negative reactions as a result of an overdose of this pathogenic factor affecting the body.

The photoplethysmographic signal is associated with the hemodynamics of the whole organism. It reflects the type of pulse wave and the connection with hemodynamics is not direct. The photoplethysmographic signal is the result of a "low pass filter" action on the pulse wave. It means that the analysis of the shape of the signal gives information about both the local blood circulation and the hemodynamics of the organism as a whole. That is why photoplethysmographs have recently been increasingly used in medical diagnostics.

Besides continuous registration, it is possible to perform an occlusion test. At the time of the development of the two-channel photoplethysmograph, there were publications about the medical use of a single-channel finger-type LED $(\lambda \approx 940 \pm 20 \mathrm{~nm})$ photoplethysmography. The use of one photosensor (one channel) with a radiation wavelength of $920 \ldots 960 \mathrm{~nm}$ will lead to "physiological interference" when registering a PPG signal. It appears because of different amounts of light absorption by the main components of blood flow - 
oxy-and deoxy-hemoglobin. That's why it is impossible to obtain acceptable accuracy, repeatability and adequacy of the PPG signal. Therefore, a new laser $(\lambda=$ $805 \pm 0.75 \mathrm{~nm}, \mathrm{P}=0.1 \ldots 0.2 \mathrm{~mW})$ two-channel twofinger photoplethysmograph has been developed. The radiation wavelength of $804.25 \ldots 805.75 \mathrm{~nm}$ is the "isobetic point" for oxy- and deoxy-hemoglobin. This is the wavelength at which the spectral characteristics of these two substances coincide. Therefore, it is possible to avoid "physiological interference" when obtaining a photoplethysmogram.

In the previously developed photoplethysmographs the individual physiological state of person was not taken into account. Such physical factors as electromagnetic radiation, radioactive radiation, electrical, magnetic, mechanical and other factors differently affect the physiological state of a person. The photoplethysmograms of different people differ due to different optical density of human tissues. In the developed two-channel photoplethysmograph, the individual physiological state of the examined worker is taken into account. Therefore the doctor receives a photoplethysmogram whith reliable information about the surveyed employee. The device also can take artificial occlusion using a conventional cuff. The device has a valve and a compressor with a pressure sensor. Occlusive photoplethysmography is widely used in the diagnosis of vascular tone.

The main technical characteristics of the FPG-2KL device [7]:

- registration, display and linear filtering of biomedical information in real time;

- support for standard (Nell-Cor-compatible) and laser photoplethysmographic sensors; synchronous registration of the photoplethysmographic signal and pressure;

- use of laser diodes to operate the device at the isobestic point $(805 \mathrm{~nm})$;

- wide dynamic range (the conversion factor of the photocurrent varies in the interval $2 \cdot 10^{5} \ldots 2 \cdot 10^{6}$ ), so the device can be used for studies of peripheral blood flow;

- two-channel operation mode. Both channels operate in parallel with a sampling rate of $350 \mathrm{~Hz}$ per channel;

- useful signal bandwidth $0.1 \ldots 40 \mathrm{~Hz}$;

- automatic artificial occlusion using a compressor and an electronic valve;

- option for the artificial occlusion level control;

- the set includes software for automated processing and analysis of registered information.

The appearance of the device is shown in Fig. 4 (conditionally named FPG-2KL - two-channel laser photoplethysmograph).

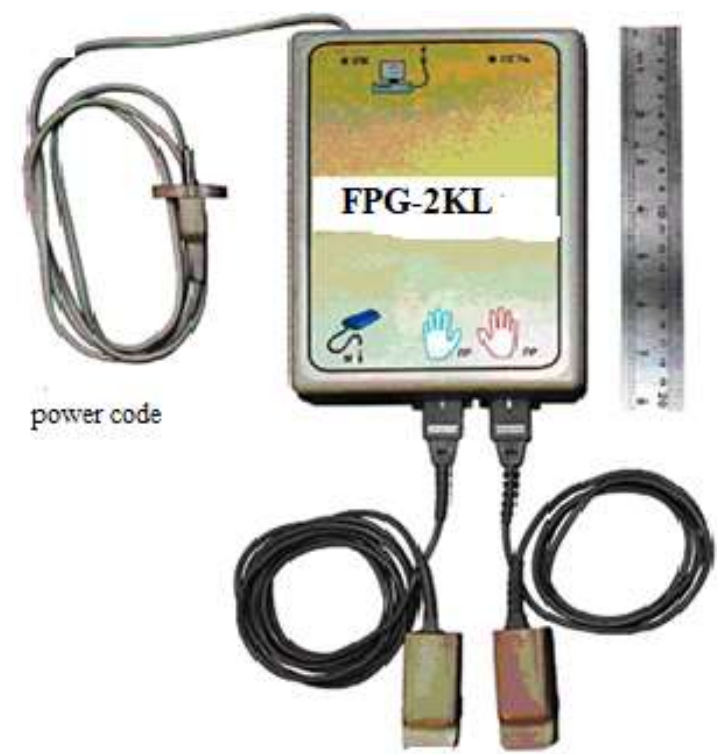

laser sensors

Fig. 4. Two-channel two-finger laser photoplethysmograph.

The block diagram of a two-channel laser photoplethysmograph is shown in Fig. 5.

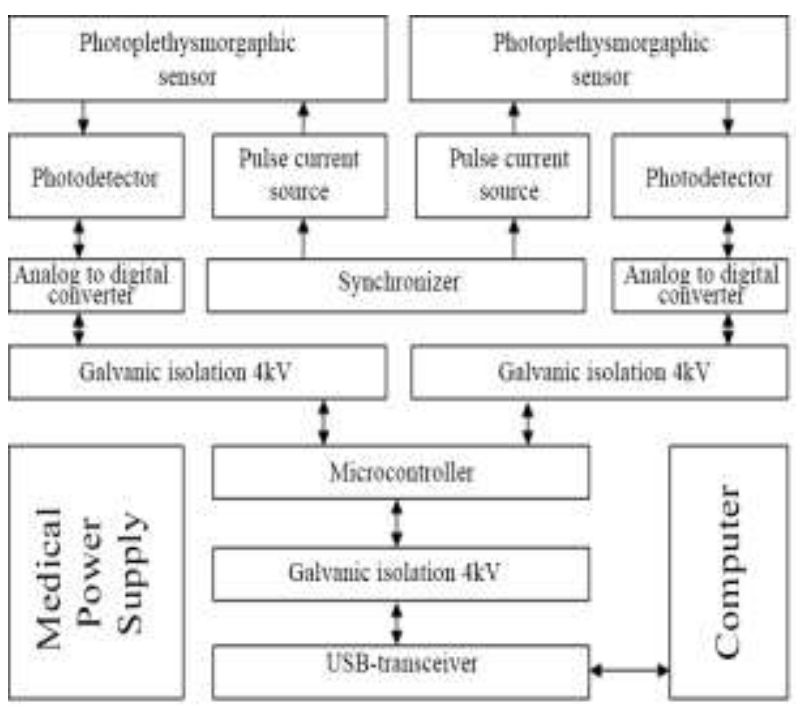

Fig. 5. Block diagram of a two-channel two-finger laser photoplethysmograph.

\section{Conclusions}

The developed digital automated medical complex for pre-shift examination (survey) of the energy complex enterprises personnel is an effective tool for medical workers. It can significantly reduce the time required for pre-shift examinations. It can reliably diagnose deviations of some physiological parameters, such as temperature, pressure, pulse and other physiological parameters. It gives a prognostic conclusion based on digital processing of obtained physiological parameters. The reliability of data obtained is based on the chosen methodology and principles of the interaction of laser radiation with the main components of blood flow. 
Medical digital precision thermometer measures an important diagnostic parameter - the temperature of the person. Together with photoplethysmogram it gives more complete and accurate assessment of the physiological state of the worker.

The main feature of the developed TMCP is a temperature measurement with high accuracy of $0.01^{\circ}$ C. It ensures the registration of even minor temperature changes for diagnostic purposes and significantly expands the field of medical thermometry. Data are automatically recorded and saved in computer.

Advantages of the photoplethysmography method, carried out in the developed FPG-2KL photoplethysmograph:

1. provides check- in of the human body reactions to external physical effects of a pathogenic nature;

2. the use of a laser radiation wavelength of $805 \mathrm{~nm}$ excludes the appearance of a "physiological interference" at the "isobestic point"; it appears because of different amounts of light absorption by the main components of blood flow - oxy-and deoxy-hemoglobin.

3. application of two-channel laser photoplethysmographic system allows to compare both signals, improve the overall quality of the PPG signal, to expand the scope of photoplethysmographs.

4. the use of a two-channel laser photoplethysmograph as a recorder of changes in the state of the body under pathogenic external influence;

5. photoplethysmogram by registering changes in dynamics of blood supply in the body can assess the effect of external pathogenic influences, can control their amounts and prevent negative effects for the body.

Clinical trials of a digital automated medical complex were carried out at the "Yarovit" Urological center in Moscow, at the 5th City clinical hospital named after Bakhrushin brothers, Moscow, at the Volga Region Academy of Sports and Tourism. Positive and adequate results in the predictive control of human physiological data in a period of 8-16 hours were obtained. We have got recommendations for using the complex in medical offices of energy enterprises for pre-shift examination of personnel.

\section{References}

1. R.G. Jackson, V.V. Luchinin (ed.), The latest sensors (M.: Technosphere, World of electronics, 133-138, 2007)

2. J.M. Zurbuchen, Precision thermometry, Measurement science conference tutorial Thermometry - fundamental and practice (2000)

3. B.H. Childs, Practical Temperature Measurement (Oxford, 2001) ISBN: 0-750-65080-X

4. J.V. Nicholas, D.R. White, Traceable Temperatures (2nd ed., Chichester: John Wiley \& Sons, LTD, 2001) ISBN: 0-471-49291-4

5. A.I. Laryushin, M.A. Galkin, R.N. Khizbullin, V.A. Novikov, A.P. Kuzmich, Measurement of the temperature response of human organs to electrolaser exposure, The world of dimensions, 3, 21-25 (2010)

6. A.I. Laryushin, M.A. Galkin, R.N. Khizbullin, V.A. Novikov, Medical digital precision thermometer, The world of dimensions, 9, 9-17 (2010)

7. A.I. Laryushin, M.A. Galkin, R.N. Khizbullin, V.A. Novikov Two-channel laser photoplethysmograph, The world of dimensions, 7, 22-28 (2010)

8. R. Khizbullin, R. Khizbullin, Ways to improve safety in the power industry: an automated hardware complex for pre-shift inspection of personnel of power enterprises, SES-2019, E3S Web of Conferences, 124, 05037 (2019)

9. R. Khizbullin, R. Khizbullin, V. Galiakhmetov, Instrument implementation of an automated complex for recording physiological parameters of drivers, KTTI-2019, E3S Web of Conferences, 157, 01005 (2020)

10. E.M. Khusnutdinova, P.P. Pavlov, V.P. Fandeyev, R.N. Khizbullin A.N. Khusnutdinov, I.V. CHerepenkin, Comprehensive Test Procedure for Digital Instruments and Devices of Automated Versatile Systems, KTTI-2019, E3S Web of Conferences, 157, 01005 (2020)

11. L.S. Sabitov, R.N. Khizbullin, R.N. Khizbullin, P.P. Pavlov, A.N. Khusnutdinov, A.R. Abdullina, Medical and Biological Problems in the Control of Therapeutic Exposure Parameters in Sports Medicine and Ways of Their Solution in New Medical Instruments and Systems, ISTC-IETEM2019 (2019)

12. R.N. Khizbullin, M.F. Zamaltdinov, Justification of the choice of the sensitive element of a precision temperature sensor, Proceedings of higher educational institutions, Problems of Energy, 1-2, 38-45 (2011)

13. R.N. Khizbullin, M.A. Galkin, V.P. Nevzorov, Evaluation of the effectiveness of manual exposure using high-resolution laser plethysmography, Manual therapy, Scientific and practical journal, 2, 42-48 (2012)

14. R.N Khizbullin, A.I. Laryushin, Automated medical hardware complex for pre-shift examination of personnel of energy enterprises, Proceedings of higher educational institutions, Problems of Energy, 1-2, 125-133 (2014). 